Regards sur l'économie allemande

Bulletin économique du CIRAC

$118-119 \mid 2015$

Varia

\title{
Réfugiés : comment les intégrer en emploi ?
}

\section{Isabelle Bourgeois}

\section{OpenEdition}

Journals

Édition électronique

URL : http://journals.openedition.org/rea/4896

DOI : $10.4000 /$ rea.4896

ISSN : 1965-0787

\section{Éditeur}

CIRAC

\section{Édition imprimée}

Date de publication : 31 décembre 2015

Pagination : 35-37

ISSN : 1156-8992

\section{Référence électronique}

Isabelle Bourgeois, «Réfugiés : comment les intégrer en emploi ?», Regards sur l'économie allemande [En ligne], 118-119 | décembre 2015, mis en ligne le 31 décembre 2017, consulté le 15 septembre 2020. URL : http://journals.openedition.org/rea/4896

Ce document a été généré automatiquement le 15 septembre 2020.

(c) CIRAC 


\title{
Réfugiés : comment les intégrer en emploi ?
}

\author{
Isabelle Bourgeois
}

1 La question de l'intégration des réfugiés figure en tête de l'agenda en Allemagne. Elle préoccupe non seulement les citoyens qui se chargent de l'accueil, les responsables politiques ou les diverses administrations concernées, mais également les milieux scientifiques qui contribuent activement au débat et à la prise de décision en publiant analyses et études.

\section{L'intégration est un long processus}

2 Ainsi, le Conseil des Sages par exemple y consacre un long développement dans son dernier rapport annuel, publié le 11 novembre 2015. Et comme il est d'usage dans toute discussion sur la manière de relever un nouveau défi, il procède à un état des lieux de l'existant avant d'établir des prévisions qui, du fait des nombreuses inconnues concernant les migrants, ne peuvent être que purement hypothétiques. Avant toute chose, le Conseil cherche donc à savoir comment se sont intégrées dans le marché du travail les différentes catégories de la société allemande, selon qu'elles ont un « arrièreplan migratoire » ou non, pour reprendre la formule consacrée employée par Destatis.

Structure de l'activité selon les origines migratoires ou non des actifs en 2014

\begin{tabular}{|c|c|c|c|c|c|c|c|c|}
\hline \multirow[t]{2}{*}{ Personnes } & \multicolumn{4}{|l|}{ Ensemble } & \multicolumn{2}{|c|}{ Hommes $15-64$ ans } & \multicolumn{2}{|c|}{ Femmes $15-64$ ans } \\
\hline & Population & $\begin{array}{l}15-64 \\
\text { ans }^{\text {a) }}\end{array}$ & $\begin{array}{l}\text { Taux } \\
\text { d'activité } \\
\text { b) }\end{array}$ & $\begin{array}{l}\text { Taux de } \\
\text { chômage } \\
\text { b) }\end{array}$ & $\begin{array}{l}\text { Taux } \\
\text { d'activité } \\
\text { b }\end{array}$ & $\begin{array}{l}\text { Taux de } \\
\text { chômage } \\
\text { b) }\end{array}$ & $\begin{array}{l}\text { Taux } \\
\text { d'activité } \\
\text { b) }\end{array}$ & $\begin{array}{l}\text { Taux de } \\
\text { chômage } \\
\text { b) }\end{array}$ \\
\hline & milliers & \multicolumn{7}{|l|}{ en $\%$} \\
\hline
\end{tabular}




\begin{tabular}{|l|l|l|l|l|l|l|l|l|}
\hline $\begin{array}{l}\text { Allemands de } \\
\text { souche }\end{array}$ & $\mathbf{6 4} 511$ & $\mathbf{6 4 , 7}$ & $\mathbf{7 9 , 4}$ & $\mathbf{4 , 3}$ & $\mathbf{8 3 , 2}$ & $\mathbf{4 , 6}$ & $\mathbf{7 5 , 6}$ & $\mathbf{4 , 1}$ \\
\hline $\begin{array}{l}\text { Avec arrière-plan } \\
\text { migratoire }\end{array}$ & $\mathbf{1 6 3 8 6}$ & $\mathbf{6 8 , 5}$ & $\mathbf{7 0 , 3}$ & $\mathbf{8 , 1}$ & $\mathbf{7 8 , 5}$ & $\mathbf{8 , 6}$ & $\mathbf{6 2 , 1}$ & $\mathbf{7 , 5}$ \\
\hline Immigrés & 10877 & 81,9 & 73,9 & 7,9 & 83,4 & 8,3 & 64,7 & 7,4 \\
\hline $\begin{array}{l}\text { Allemands rapatriés } \\
\text { (Spätaussiedler) c) }\end{array}$ & 5866 & 83,2 & 69,3 & 9,5 & 81,2 & 9,7 & 57,5 & 9,5 \\
\hline $\begin{array}{l}\text { Allemands } \\
\text { naturalisés }\end{array}$ & 3099 & 78,0 & 81,3 & 5,7 & 86,1 & 6,6 & 76,7 & 4,9 \\
\hline $\begin{array}{l}\text { Avec ascendants } \\
\text { immigrés }\end{array}$ & 1912 & 84,0 & 77,0 & 7,0 & 86,2 & 6,9 d) & 68,3 & 6,5 d) \\
\hline Total & $\mathbf{8 0 ~ 8 9 7}$ & $\mathbf{6 5 , 5}$ & $\mathbf{7 7 , 5}$ & $\mathbf{5 , 1}$ & $\mathbf{8 2 , 2}$ & $\mathbf{5 , 4}$ & $\mathbf{7 2 , 7}$ & $\mathbf{4 , 7}$ \\
\hline
\end{tabular}

a) part dans la population totale ; b) part parmi les $15-64$ ans ; c) Allemands de souche ayant quitté les pays de l'ex-bloc soviétique après le 01-01-1993; d) 25 à 64 ans.

Source : Sachverständigenrat zur Begutachtung der gesamtwirtschaftlichen Entwicklung, Jahresgutachten 2015/16, page 246.

3 Cette succincte photographie de l'année 2014, prise juste avant la grande vague d'immigration de cette année, montre que l'insertion dans le marché du travail prend du temps, comme le révèle la situation des descendants d'immigrés. Les handicaps sont en effet nombreux : à l'analphabétisme de certains s'ajoutent l'absence de connaissance de la culture du pays d'accueil, le manque de maittrise de la langue allemande, et le problème des qualifications qui, lorsqu'elles existent, sont rarement adaptées à la demande.

\section{Première étape : emplois atypiques}

4 Au début, donc, le temps que les nombreux cours d'alphabétisation, d'intégration ou d'apprentissage de la langue, ainsi que les multiples mesures de pré-qualification déploient leurs effets, les réfugiés seront confrontés à toutes les formes classiques du chômage d'insertion. Puis, leur insertion progressive en emploi se traduira par une hausse du nombre de stages et d'emplois atypiques - des mini-jobs à l'intérim. Ceux-ci présentent un double avantage: aux réfugiés, ils permettent de «mettre le pied à l'étrier ", voire de faire leurs preuves ; aux employeurs, ils offrent "le degré de flexibilité requis face à l'incertitude quant à la productivité des demandeurs d'emploi ", résume le Conseil des Sages.

\section{Le SMIC légal est un handicap}

5 Dans ce contexte, le salaire minimum légal entré en vigueur au $1^{\mathrm{er}}$ janvier de cette année se révèle "une forte barrière à l'insertion en emploi » des réfugiés, estime le Conseil des Sages, qui préconise dès lors de porter la durée de sa non-application aux chômeurs 
de longue durée de 6 à 12 mois, tout en recommandant plus généralement de ne le revaloriser à aucun prix pour ne pas étouffer la demande d'emplois qui va s'accroître dans le segment à bas salaires.

6 Au total donc, au cours des prochaines années, le nombre de demandeurs d'emploi devrait être légèrement en hausse, et parmi eux, celui des bénéficiaires du régime d'assistance «Hartz $I V$ » qui permet de cumuler le revenu issu d'un mini-job avec les aides forfaitaires destinées au demandeur d'emploi et aux membres de sa famille.

\section{Le « devoir humanitaire » qu'est l'intégration a un coût}

7 Quant au coût de l'intégration des réfugiés, le Conseil des Sages l'estime dans une fourchette de 5,9 à 8,3 milliards $€$ cette année (soit entre 0,2 et $0,3 \%$ du PIB), puis de 9 à 14,3 milliards $€$ en 2016 (de l'ordre de 0,3 à 0,5\% du PIB). Dans leur rapport d'automne, le groupe de travail des instituts économiques, qui se penche lui aussi sur la question, estime ce coût à environ 4 milliards $€$ cette année, puis à 11 milliards $€$ l'an prochain. La différence s'explique en grande partie par le fait que le rapport des instituts est paru début octobre, celui du Conseil des Sages en novembre, ce qui a permis à ce dernier d'intégrer des données actualisées. Tous deux n'en rappellent pas moins que si l'accueil des réfugiés pèse actuellement sur les budgets publics, il s'agit d'un «devoir humanitaire" pour l'Allemagne. Certes, l'arrivée des réfugiés ne remplace pas une politique ciblée d'immigration qualifiée, elle n'en présente pas moins une opportunité à saisir pour une société vieillissante.

\section{Qui sont les réfugiés ? Et combien resteront ?}

Encore faut-il mieux connaître cette nouvelle population pour bien l'intégrer. Elle est difficile à chiffrer : il y a d'une part ceux qui entrent sur le territoire allemand et dont le nombre devrait atteindre 1 million sur l'année 2015, mais tous ne sont pas encore recensés ni enregistrés. Quoi qu'il en soit, entre janvier et août 2015, le nombre de réfugiés arrivés en Allemagne a atteint 414000 selon les données du Système EASY (Erstverteilung von Asylbegehrenden) qui répertorie notamment la nationalité des nouveaux arrivés et les répartit dans les centres d'accueil. Pour sa part, l'office fédéral pour la migration et les réfugiés (BAMF), qui gère entre autres les demandes d'asile, a enregistré au cours de la même période 231000 demandes d'asile. Du fait de ces seules différences dans les missions de ces institutions, nul ne connaît actuellement le nombre exact de réfugiés ou demandeurs d'asile, et nul ne sait donc encore combien, sur le million de réfugiés, resteront à terme en Allemagne; tout au plus les hypothèses situent-elles généralement ce nombre aux alentours de 300 000, voire plus; mais ces chiffres sont à prendre avec précaution.

Les dix principaux pays d'origine des migrants de 2012 à la fin du $1^{\mathrm{er}}$ semestre 2015 (dépôts de demande d'asile, $7^{\text {re }}$ étape)

\begin{tabular}{|l|l|l|l|l|l|l|l|l|}
\hline Pays et rang & & $\mathbf{2 0 1 2}$ & & $\mathbf{2 0 1 3}$ & & $\mathbf{2 0 1 4}$ & $\begin{array}{l}\text { Sem. } \\
\mathbf{1 / 2 0 1 5}\end{array}$ \\
\hline Serbie & 1 & 8477 & 3 & 11459 & 2 & 39332 & $\mathbf{4}$ & 10124 \\
\hline
\end{tabular}




\begin{tabular}{|l|l|l|l|l|l|l|l|l|}
\hline Afghanistan & 2 & 7498 & 4 & 7735 & 4 & 9115 & 6 & 21806 \\
\hline Syrie & 3 & 6201 & 2 & 11851 & 1 & 39332 & 1 & 32472 \\
\hline Irak & 4 & 5352 & 8 & 3958 & 10 & 5345 & 5 & 8331 \\
\hline Macédoine & 5 & 4546 & 5 & 6208 & 8 & 5614 & 7 & 4182 \\
\hline Iran, Rép. Islam. & 6 & 4348 & 6 & 4424 & & - & & - \\
\hline Pakistan & 7 & 3412 & 7 & 4101 & & - & 10 & 2701 \\
\hline Fédération de Russie & 8 & 3202 & 1 & 14887 & & - & & - \\
\hline Bosnie Herzégovine & 9 & 2025 & - & 7 & 5705 & & - \\
\hline Kosovo & 10 & 1906 & - & 6 & 6908 & 2 & 28672 \\
\hline Total des 10 premiers pays & & $\mathbf{4 6 9 6 7}$ & 72025 & $\mathbf{1 1 5} 782$ & $\mathbf{1 2 2} 609$ \\
\hline Total des demandes d'asile & & 64539 & 109580 & 173072 & & 159927 \\
\hline $\begin{array}{l}\text { Part des 10 premiers pays dans le total } \\
\text { des demandes d'asile }\end{array}$ & & $72,8 \%$ & $\mathbf{6 5 , 7} \%$ & $\mathbf{6 6 , 9} \%$ & $76,7 \%$ \\
\hline
\end{tabular}

Source : Bundesamt für Migration und Flüchtlinge (BAMF), Schlüsselzahlen Asyl 1. Halbjahr 2015, août 2015 (www.bamf.de).

9 L'origine des réfugiés s'est beaucoup modifiée depuis le début de l'année, explique l'institut IAB qui consacre une étude aux migrants et réfugiés sur le marché du travail allemand (Aktuelle Berichte, $\mathrm{n}^{\circ}$ 14/2015). Selon les données communiquées par le Système EASY, 53 \% des nouveaux arrivés au cours des huit premiers mois de l'année viennent de pays où règnent guerre, guerre civile ou répression politique, $31 \% \mathrm{de}$ l'Est des pays du Balkan. Mais en août, la part des premiers est montée à $76 \%$, celle des seconds tombant à $12 \%$. Les seuls Syriens représentaient alors près de la moitié (44 \%) des nouveaux réfugiés.

\section{Le droit d'asile est un droit fondamental}

10 Ces derniers devraient se voir accorder presque automatiquement le droit d'asile, indépendamment de l'entrée en vigueur, le 24-10-2015, de la Loi sur l'accélération des procédures d'asile (Asylverfahrensbeschleunigungsgesetz). En effet, l'art. 16a de la Loi fondamentale accorde le droit d'asile à toute victime de persécutions politiques. C'est là le seul droit fondamental de la Constitution allemande s'appliquant exclusivement aux étrangers - et ce, dans le respect du premier droit fondamental, celui de la dignité humaine (art. 1 de la Loi fondamentale). C'est peut-être là aussi qu'il faut chercher l'attractivité qu'exerce l'Allemagne sur les réfugiés politiques, dans un droit d'asile dont l'acception dépasse de loin celle de la Convention relative au statut des réfugiés, signée à Genève en 1951. 


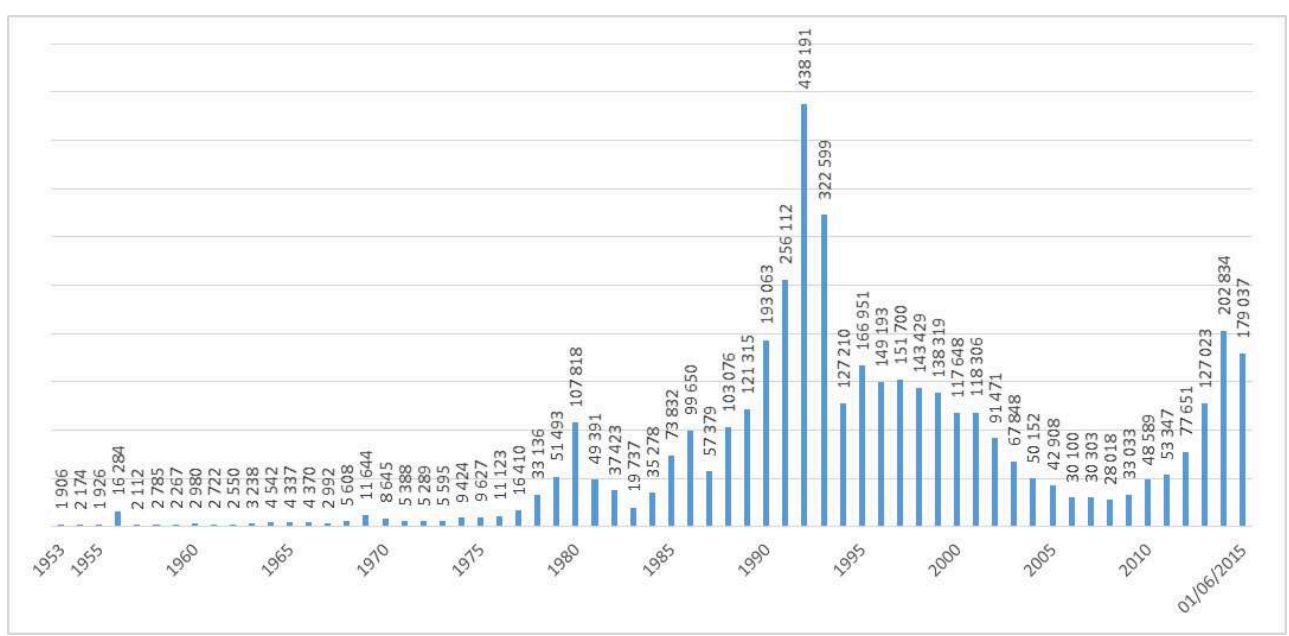

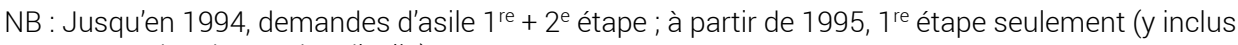
réexamens des demandes d'asile).

Source : Bundesamt für Migration und Flüchtlinge (BAMF), Schlüsselzahlen Asyl 1. Halbjahr 2015, août 2015 (www.bamf.de).

\section{Faible niveau de qualification et jeunesse des réfugiés}

Quant au niveau de qualification des réfugiés, s'il n'est guère connu non plus pour l'instant avec précision, les premières estimations de l'Agence fédérale pour l'emploi de Nuremberg révèlent que si, globalement, le niveau d'études des réfugiés semble nettement supérieur à leur niveau de qualification professionnelle, la grande majorité d'entre eux n'a pas achevé de scolarité. Une difficulté dans l'appréciation des qualifications réside notamment dans le fait que la grille d'évaluation de l'OCDE, si elle est utile pour effectuer des comparaisons internationales à l'échelon macroéconomique, ne se révèle guère adaptée à la praxis, et tout particulièrement aux pratiques nationales respectives. Il est vrai aussi, explique l'institut IAB, que $55 \%$ d'entre eux ont moins de 25 ans ; parmi les réfugiés arrivés en 2014, on compte $28 \%$ d'enfants de 0 à 15 ans.

\section{Priorité donc à la formation, clé de l'insertion}

Tout cela pose un sérieux défi au système d'éducation et de formation professionnelle. Et de fait, les initiatives les plus diverses se multiplient, que ce soient les entreprises qui proposent des stages de pré-qualification ou des places d'apprentissage, voire des cours d'allemand en sus, ou que ce soient les établissements du système scolaire, avec toute la difficulté que représente l'impératif de respecter une grande mixité en termes d'origines pour ne pas créer des ghettos. Quoi qu'il en soit, après l'urgence de trouver un toit aux réfugiés, il faut maintenant les qualifier pour que ceux qui resteront s'intègrent avec succès dans le marché du travail et donc la société allemande. A cet égard, la nomination le 18-09-2015 de Frank-Jürgen Weise, Président du directoire de l'Agence fédérale pour l'emploi, à la tête de l'office fédéral pour la migration et les réfugiées - postes qu'il occupe parallèlement -, pourrait se révéler de bon augure. 
INDEX

Mots-clés : réfugiés, emploi, intégration, formation 